\title{
Immunohistochemistry of Ecchordosis Physaliphora and Chordoma
}

\author{
R.L. Macdonald and J.H.N. Deck
}

\begin{abstract}
Immunohistochemical stains for cytokeratins, vimentin, epithelial membrane antigen, carcinoembryonic antigen and S-100 were performed on nine ecchordoses and 10 chordomas. $100 \%$ of the ecchordoses were positive for cytokeratin, vimentin and epithelial membrane antigen. $78 \%$ were $S-100$ positive. $100 \%$ of the chordomas stained positively for cytokeratin, vimentin and S-100 and $90 \%$ were positive for epithelial membrane antigen. No ecchordosis or chordoma was carcinoembryonic-antigen positive. These findings are interesting for two reasons. First, the similar staining properties of chordoma and ecchordosis physaliphora support the embryologic hypothesis of their common origin from the fetal notochord. Second, they indicate that when an ecchordosis causes symptoms prompting surgical resection, differentiation of the lesion from chordoma with routine immunohistochemistry would not be helpful.

RÉSUMÉ: Immunohistochimie de l'ecchordose physaliphore et du chordome Nous avons exécuté des colorations immunohistochimiques pour les cytokératines, la vimentine, l'antigène épithélial membranaire, l'antigène carcinoembryonnaire et le S-100 sur neuf ecchordoses et dix chordomes. Cent pourcent des ecchordoses étaient positifs pour la cytokératine, la vimentine et l'antigène épithélial membranaire. Soixante-dix-huit pourcent étaient positifs pour le S100. Cent pourcent des chordomes étaient positifs pour la cytokératine, la vimentine et le S-100 et quatre-vingt-dix pourcent étaient positifs pour l'antigène épithélial membranaire. Aucun des ecchordoses ou des chordomes n'était positif pour l'antigène carcino-embryonnaire. Ces observations sont intéressantes pour deux raisons. D'abord, les propriétés histochimiques similaires des chordomes et des ecchordoses supportent l'hypothèse embryologique de leur origine commune à partir de la notocorde foetale. Ensuite, elles indiquent que, lorsqu'un ecchordose provoque des symptômes qui motivent une résection chirurgicale, il ne serait pas coloration immunohistochimique.
\end{abstract}

Can. J. Neurol Sci. 1990; 17: 420-423

The fetal notochord is an interesting embryologic structure which is postulated to give rise to normal, vestigial and pathologic structures in the adult. In the human, the notochord forms during the third week of embryonic life. ${ }^{1}$ It is important in the genesis of the axial skeleton and normally persists in the adult as the nucleus pulposus of the intervertebral disc. 2,3 Several other remnants of the notochord may also persist into adulthood. Heterotopic rests of notochord cells may be found outside the nucleus pulposus anywhere along the axial skeleton from the coccyx to the dorsum sella.2,4 They are most common at the extreme ends of the skeleton. Chordomas are felt to arise from these ectopic rests of notochordal cells and, similar to the cell rests, they are extradural in location and most common in the sacrum or clivus. ${ }^{3.5-7}$ The prognosis remains dismal with a median survival of five years from the time of diagnosis. $3,5,6,8$ The pathological features of chordoma have been extensively studied.5-19

Another structure which is thought to be derived from the notochord and which has received less attention in the pathology literature is the ecchordosis physaliphora (Figures 1 and 2).11,20-25
These jelly-like excrescences are usually related to the clivus but may be found anywhere along the axial skeleton.2,4,21 Ecchordoses are considered to be non-neoplastic hamartomas which are clinically silent lesions, and rarely, if ever, are the source of chordomas. ${ }^{3,8}$ However, there are a number of reports of symptomatic ecchordoses and of patients with symptoms due to intradural lesions composed of physaliphorous cells which have all the gross features of ecchordoses although they have been larger than the usual ecchordosis. ${ }^{1,21,26-28} \mathrm{~A}$ recent case of what we diagnosed as symptomatic ecchordosis led us to attempt to differentiate the lesion from chordoma, which would certainly carry a more grave prognosis. ${ }^{29}$ This paper reports the results of immunohistochemical staining of ecchordosis physaliphora and chordoma. A brief discussion follows concerning the pathologic and embryologic implications of these results.

\section{Materials and Methods}

The surgical and autopsy pathology files of the Department of Pathology at Toronto General Hospital were searched for all

From the Division of Neuropathology, Department of Pathology, University of Toronto, Toronto General Hospital, Toronto

Received March 1, 1990. Accepted in final form August 16, 1990

Reprint requests to: J.H.N. Deck, Department of Pathology, Toronto General Hospital, 200 Elizabeth Street, Toronto, ON Canada M5G 2 C4 


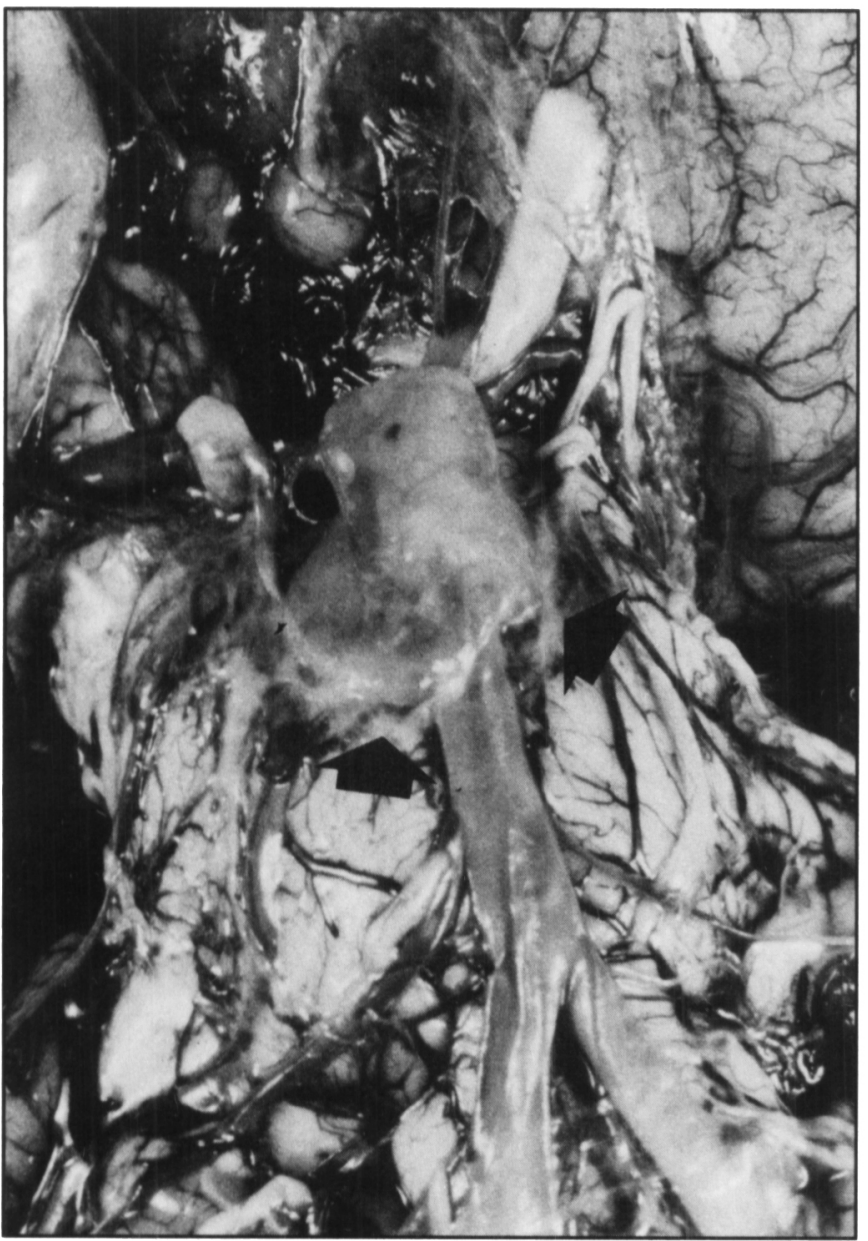

Figure I - A photograph of the ventral surface of the brainstem showing a gelatinous ecchordosis physaliphora (arrow's) adherent to the arachnoid overlying the pons.

specimens diagnosed as chordoma and ecchordosis physaliphora over the years 1960 to 1988 . Specimens labelled as ecchordoses were all, with one exception, typical gelatinous excrescences attached to the dorsal clivus whose presence was disclosed only at autopsy. The exception was a lesion with the histologic appearance of an ecchordosis which was discovered spanning the clivus from sphenoid sinus to the prepontine cistern resulting in a cerebrospinal fluid fistula in a 66-year-old woman. ${ }^{29}$ The chordomas were sacrococcygeal or clival tumors diagnosed as chordomas on the basis of typical light and, occasionally, electron microscopy. Material for light microscopy was fixed in $10 \%$ formalin and embedded in paraffin. Sections of the same blocks were stained immunohistochemically for cytokeratins (CK, Hybritech pooled monoclonal antibodies to human epithelial keratins and Dako rabbit polyclonal anti-keratin), epithelial membrane antigen (EMA, Dako), S-100 (Dako), Vimentin (Histogen) and carcinoembryonic antigen (CEA, Dako). Positive controls were derived from standard blocks known to contain the appropriate antigens.

The results of staining were read as focally or diffusely positive or as negative. Classification of the particular lesion as diffusely or focally positive was made on a subjective basis. However, most samples were either obviously diffusely positive

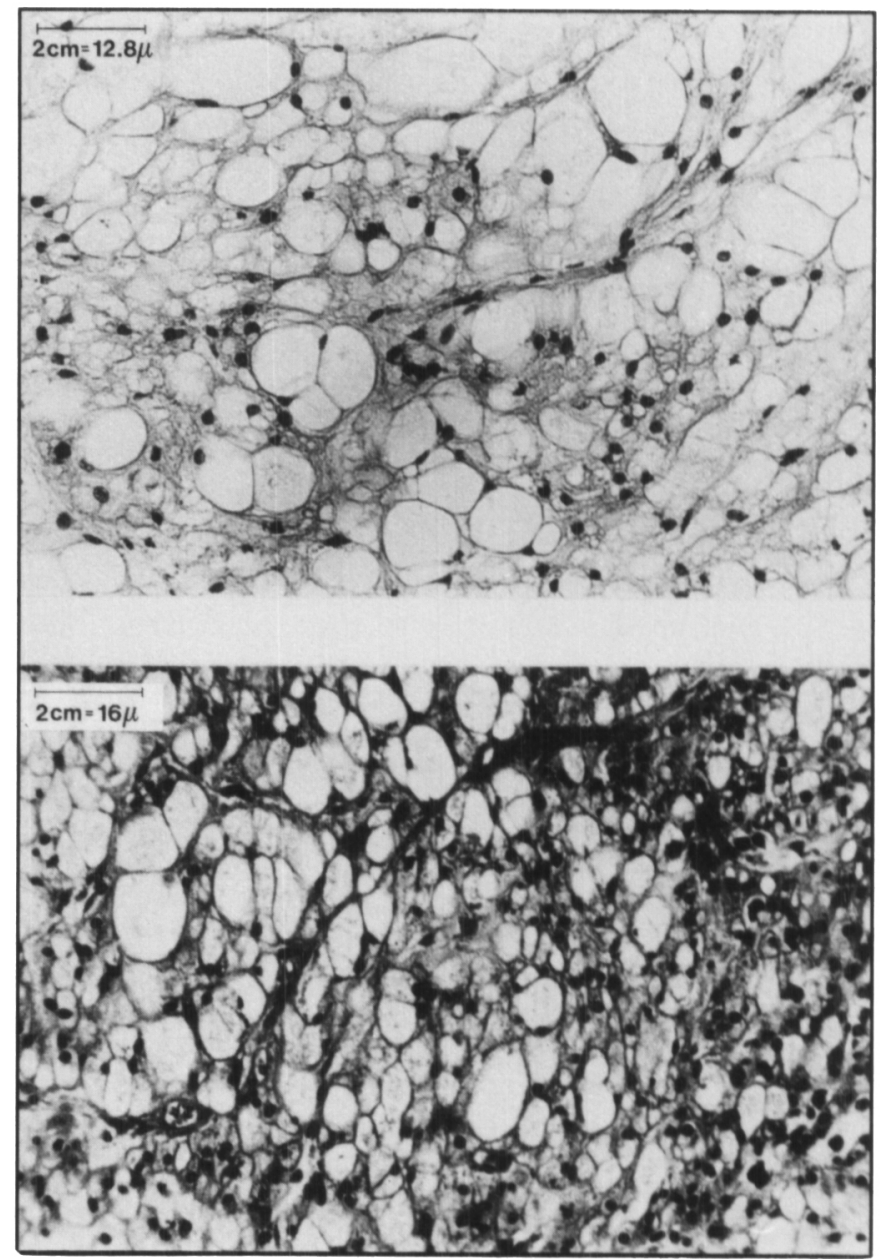

Figure 2 - Photomicrographs of an ecchordosis physaliphora (upper) and of a chordoma (lower) shouing prominent physaliphorous cells in both lesions. The chordoma is more cellular and pleomorphic than the ecchordosis. Hematoxylin and eosin.

or entirely negative; patchy and irregular staining specimens were uncommon.

\section{Results}

Figure 2 shows the typical light microscopic histology of ecchordosis physaliphora and chordoma. On light microscopy, both types of lesions contained typical physaliphorous cells with pools of PAS positive intra- and extracellular mucin. The ecchordoses were generally less pleomorphic and cellular than the chordomas although an attempt to quantify this difference was not made.

The results of immunohistochemical staining of 10 chordomas and nine ecchordoses are shown in Table 1 and Figure 3. Focal or diffuse positive staining is recorded as positive in the table. Staining with monoclonal and polyclonal CK stains produced identical results, hence the single CK column in the table. All the ecchordoses stained diffusely positive with CK. Similarly $90 \%$ of the chordomas were diffusely positive. A single chordoma was only focally positive. Vimentin was diffusely 
Table 1: Table showing the number of specimens staining positively for the various immunohistochemical markers

$\begin{array}{lrccccc} & \text { Cytokeratins } & \text { Vimentin } & \text { EMA } & \text { CEA } & \text { S-100 } \\ \text { Lesion } & 9 & 9 & 9 & 0 & 8 \\ \text { Ecchordosis }(\mathrm{N}=9) & 10 & 10 & 9 & 0 & 10 \\ \text { Chordoma }(\mathrm{N}=10) & & & & & \end{array}$

positive in all cases. All the ecchordoses were EMA positive whereas only nine chordomas were positive, seven of them diffusely and two focally. No specimen showed CEA reactivity. Positive diffuse staining with S-100 was observed in seven ecchordoses and 10 chordomas. One ecchordosis was focally positive for S-100 and one was negative.

\section{Discussion}

Small gelatinous masses of tissue applied to the dorsal aspect of the clivus were first described by Luschka in $1856 .{ }^{30}$ These are now recognized as ecchordosis physaliphora (Figure 1) ${ }^{3.24}$ A light microscopic description was supplied in 1857 by Virchow who utilized the term ecchondrosis physaliphora sphe. nooccipitalis, believing they were derived from the cartilage of the sphenooccipital synchondrosis. ${ }^{31}$ With the initial suggestion by Muller (1858) and subsequent experimental evidence provided by Ribbert (1894) consensus has arisen that ecchordoses are embryologic relics of the fetal notochord. 22,23 Light and electron microscopic data confirm structural similarity between fetal notochord and ecchordosis physaliphora. Both are composed of physaliphorous cells. ${ }^{11.20}$ The electron microscopic characteristics are almost identical and include intracytoplasmic vacuoles, intermediate filaments, plasmalemmal invaginations and endoplasmic reticulum-mitochondria complexes. 11.20,25

Chordomas also share histologic and ultrastructural features with both ecchordosis physaliphora and fetal notochord (Figures 2 and 3). Chordomas contain physaliphorous cells and on electron microscopy have the same features as notochord and ecchordosis physaliphora.6.7.11.16.18 Immunohistochemical staining has been reported for chordoma and notochord. As one would expect, their characteristics are similar. ${ }^{10.17}$ Both tissues express CK, EMA, vimentin and S-100.9.12-15,17.32.33 There are few reports of differences between these tissues although Bottles and Beckstead found $5^{\prime}$-nucleotidase activity on the plasma membrane of chordoma and not on fetal notochord. ${ }^{34}$

The immunohistologic features of ecchordoses have not been previously reported but our results using routine immunohistochemistry confirm that ecchordoses, like chordomas, express CK, EMA, S-100 and vimentin (Figure 3). These findings provide further support for the embryologic derivation of ecchordoses from the fetal notochord. 2,3,23

These results are important because of the occasional need to differentiate pathologically between chordoma and ecchordosis physaliphora. A number of cases of symptomatic ecchordoses which may be pathologically similar to chordomas have been reported.21.26-29 Some authors have used the term intradural or benign chordoma for such ecchordoses. 1.5.27 Unlike true chordomas, however, these cases have presented with apoplexy and only rarely with compressive neurologic symptoms. The prognosis of symptomatic ecchordosis is better than for chordoma as they are surgically resectable and they do not invade bone. Hence the need for pathologic distinction. ${ }^{1}$ However, routine

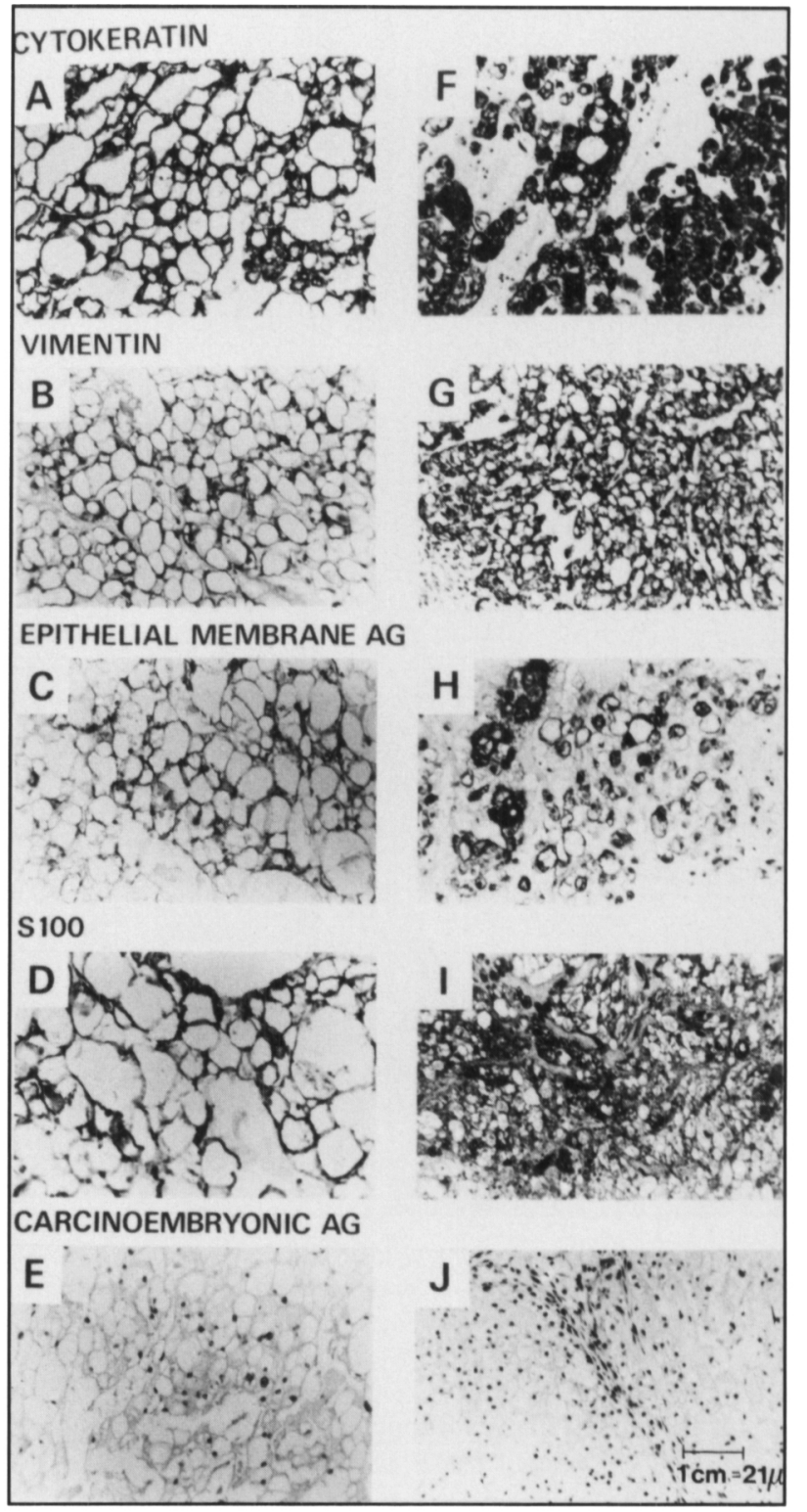

Figure 3-Photomicrographs showing the immunohistochemical staining of ecchordosis physaliphora (left. A to E) and chordoma (right. $F$ to J). Both lesions express cytokeratin ( $A$ and $F$ ), vimentin ( $B$ and $G)$, epithelial membrane antigen ( $C$ and $H)$ and $S-I 00(D$ and $I)$ as show'n by the dark staining. There is no positive staining with carci. noembryonic antigen ( $E$ and $J$ ). ( $A G$; antigen. The calibration bar on photomicrograph J applies to all photomicrographs in this illustration).

light and electron microscopy and, from this study, immunohistochemistry, fail to aid in this distinction.

At present, the diagnosis of symptomatic ecchordosis must rest on the clinical and radiologic features combined with a consistent pathologic lesion. In the future new markers and diagnostic methods may aid in distinguishing chordoma and ecchordosis physaliphora.

\section{ACKNOWLEDGEMENTS}

The authors thank Sheilah Macdonald and Arlene McLean for assistance in preparation of this manuscript. We also thank Karen Popowich and Sam Motyko for help in preparing the illustrations. 


\section{REFERENCES}

1. Wolfe JT, Scheithauer BW. "Intradural chordoma" or "giant ecchordosis physaliphora"? Report of two cases. Clin Neuropathol 1987; 6: 98-103.

2. Horwitz T. Chordal ectopia and its possible relation to chordoma. Arch Pathol 1941; 31: 354-362.

3. Sassin JF. Intracranial chordoma. In: Vinken PJ, Bruyn GW, eds. Handbook of Clinical Neurology. Vol. 18, Amsterdam: NorthHolland 1975; 151-164.

4. Ulich TR, Mirra JM. Ecchordosis physliphora vertebralis. Clin Orthop 1982; 163: 282-289.

5. Congdon CC. Benign and malignant chordomas: a clinicoanatomical study of twenty-two cases. Am J Pathol 1952; 28: 793-821.

6. Rich TA, Schiller A, Suit HD, et al. Clinical and pathologic review of 48 cases of chordoma. Cancer 1985; 56: 182-187.

7. Rutherfoord GS, Davies AG. Chordomas - ultrastructure and immunohistochemistry: a report based on the examination of six cases. Histopathol 1987; 11: 775-787.

8. Dahlin DC, MacCarty CS. Chordoma: a study of fifty-nine cases. Cancer 1952; 5: 1170-1178.

9. Abenoza P, Sibley RK. Chordoma: an immunohistologic study. Hum Pathol 1986; 17: 744-747.

10. Burger PC, Makek M, Kleihues P. Tissue polypeptide antigen staining of the chordoma and notochordal remnants. Acta Neuropathol 1986; 70: 269-272.

11. Ho K-L. Ecchordosis physaliphora and chordoma: a comparative ultrastructural study. Clin Neuropathol 1985; 4: 77-86.

12. Coindre JM, Rivel J, Trojani M, et al. Immunohistological study in chordomas. J. Pathol 1986; 150:61-63.

13. Kontozoglou T, Qizilbash AH, Sianos J, et al. Chordoma: cytologic and immunocytochemical study of four cases. Diagn Cytopathol 1986; 2 : 55-61.

14. Miettinen M, Lehto VP, Dahl D, et al. Differential diagnosis of chordoma, chondroid, and ependymal tumors as aided by antiintermediate filament antibodies. Am J Pathol 1983; 112: 160-169.

15. Nakamura Y, Becker LE, Marks A. S-100 protein in human chordoma and human and rabbit notochord. Arch Pathol Lab Med 1983; 107: 118-120.

16. Pena CE, Horvat BL, Fisher ER. The ultrastructure of chordoma. Am J Clin Pathol 1970; 53: 544-551.

17. Salisbury JR, Isaacson PG. Demonstration of cytokeratins and an epithelial membrane antigen in chordomas and human fetal notochord. Am J Surg Pathol 1985; 9: 791-797.

18. Spjut HJ, Luse SA. Chordoma: an electron microscopic study. Cancer 1964: 17: 643-656.
19. Volpe R, Mazabraud A. A clinicopathologic review of 25 cases of chordoma (a pleomorphic and metastasizing neoplasm). Am J Surg Pathol 1983; 7: 161-170.

20. Horten BC, Montague SR. Human ecchordosis physaliphora and chick embryonic notochord: a comparative electron microscopic study. Virchows Arch (Pathol Anat) 1976; 371: 295-303.

21. Kurokawa H, Miura S, Goto T. Ecchordosis physaliphora arising from the cervical vertebra, the CT and MRI appearance. Neuroradiology 1988: 30: 81-83.

22. Muller $\mathrm{H}$. Uber das vorkommen von Resten der Chorda dorsalis bei Menschen nach der Geburt, und uber ihr Verhaltnis zu den Gallertgeschwulsten am Clivus. Z Rat Med 1858; 2: 202-229.

23. Ribbert H. Uber die Ecchondrosis Physalifora Sphenooccipitalis. Zentralbl Allg Pathol Pathol Anat 1894; 5: 457-461.

24. Stewart MJ, Burrow JLF. Ecchordosis physaliphora sphenooccipitalis. J Neurol Psychopathol 1923; 4: 218-220.

25. Wyatt RB, Schochet SS Jr, McCormick WF. Ecchordosis physaliphora: an electron microscopic study. J Neurosurg 1971: 34: 672-677.

26. Bartolini G. Il cordoma del clivus quale causa di emmoragia cerebrale: discussione anatomo-clinica su di una osservazione autoptica. Boll Soc Ital Biol Sper 1974; 50: 912-918.

27. Mapstone TB, Kaufman B. Ratcheson RA. Intradural chordoma without bone involvement: nuclear magnetic resonance (NMR) appearance; case report. J Neurosurg 1983; 59: 535-537.

28. Stam FC, Kamphorst W. Ecchordosis physaliphora as a cause of fatal pontine hemorrhage. Eur Neurol 1982; 21: 90-93.

29. Macdonald RL, Cusimano RD, Deck JHN, et al. Cerebrospinal fluid fistula secondary to ecchordosis physaliphora. Neurosurgery 1990; 26: 515-519.

30. Luschka $\mathrm{H}$. Die Altersveranderungen der Zwischenwirbelknorpel. Virchows Arch Pathol Anat Physiol 1856; 9: $311-327$.

31. Virchow R. Untersuchungen uber die Entwickelung des Schadelgrundes im Gesunden und Krankhaften zur Tande und uber den Einfluss Derselbe auf Schadelform, Gesichtsbildung und Gehirnbau. Berlin: G Reimer 1857; 47.

32. Krech R, Loy V, Iglesias J, et al. Immunohistologische charakterisierung von chordomen. Pathologe 1987; 8: 207-212.

33. Stosiek P, Kasper M, Karsten U. Expression of cytokeratin and vimentin in nucleus pulposus cells. Differentiation 1988; 39: 78-81.

34. Bottles K, Beckstead JH. Enzyme histochemical characterization of chordomas. Am J Surg Pathol 1984; 8: 443-447. 